\title{
The three-way interplay of motor coordination, body fatness and physical activity in adolescents: a preliminary study
}

\author{
Daniel das Virgens Chagas, Luiz Alberto Batista \\ Laboratory of Biomechanics and Motor Behavior, Institute of Physical Education and Sports, Rio de Janeiro State \\ University, Rio de Janeiro, Brazil
}

\section{Summary}

Study aim: The purposes of this study were to analyze: the influence of physical activity level on the association between motor coordination and body fatness; the influence of body fatness on the association between motor coordination and physical activity; and how much physical activity and body fatness, separately and together, explain motor coordination in adolescent boys. Material and methods: Fifty-one boys $(\mathrm{n}=51)$, aged between 12 and 14 years, participated in the study. Motor coordination and physical activity and body fat percentage were assessed with, respectively, the Körperkoordinationstest für Kinder (KTK) and a validated self-administered questionnaire (PAQ-C). Skinfold thickness was measured to assess the participant's body fat percentage. The Kruskal-Wallis test was performed to compare levels of motor coordination, physical activity, and body fat according to age $(12,13,14$ years). Bivariate and partial correlations were used to analyze the interrelationships among the selected variables.

Results: In the total studied group ( $\mathrm{n}=51$ boys), motor coordination was positively associated with physical activity level $(\mathrm{r}=0.300)$ and negatively associated with body fatness $(\mathrm{r}=-0.297)$. However, these associations were not statistically significant $(\mathrm{p}>0.05)$ when controlled for body fatness and physical activity, respectively. Also, body fat and physical activity together explained $11 \%$ of the variance in the motor coordination level in adolescent boys.

Conclusions: These findings suggest that body fatness can influence the association between motor coordination and physical activity as well as that physical activity level can influence the association between motor coordination and body fat percentage. Also, body fat and physical activity together, but not separately, partially explained the variance in the motor coordination level. Therefore, body fat and physical activity seem to be complementarily associated with motor coordination in adolescent boys.

\section{Key words: Motor competence - Body fat percentage - Adolescence}

\section{Introduction}

It is known that adequate motor coordination ability is not only related to children's general development [8], but it is also associated with some health-related gains, such as levels of body fat and physical activity [4]. In this sense, evidence suggests that motor coordination level is negatively associated with body fat percentage $[1,6,10]$ and positively associated with physical activity level $[11,14,17,21]$ in children and adolescents. Therefore, it is expected that children and adolescents with a higher body fat percentage and/ or lower physical activity level will have poorer motor coordination than their peers with a lower body fat percentage and/or higher physical activity level.
Although there is a growing body of knowledge about the associations between motor coordination level and health-related attributes, some specific issues in this interrelationship need to be better explored. Overall, evidence is limited concerning the three-way interplay of motor coordination, body fat and physical activity in children and adolescents. Indeed, most of the evidence about the topic has been obtained based on bivariate analyses between motor coordination and health-related attributes [5], which may have biased the findings and interpretations.

It is important to highlight having elevated levels of body fatness and physical activity are not mutually exclusive conditions. In fact, in practice we can see that not all individuals with a high body fat percentage display a low motor coordination level and/or are physically inactive. Thus, it 
seems plausible to consider the hypothesis that the physical activity level can influence the association between levels of motor coordination and body fatness, as well as that the levels of body fatness can influence the association between levels of motor coordination and physical activity.

Morrison et al. [16] found that the body fat percentage influenced the association between levels of motor coordination and physical activity in girls, but not in boys. Chagas and Batista [3], analyzing a mixed sample of adolescent boys and girls, found that the physical activity level can influence the association between motor coordination level and body mass index. In another study, Chagas and Batista [5] found that the associations between motor coordination and physical activity, as well as between motor coordination and body fat percentage, were not influenced by body fat percentage and physical activity level, respectively, in adolescent girls.

Literature about the topic is, therefore, controversial and scarce, mainly concerning adolescent boys. Thus, the purposes of this study were:

1) to analyze the influence of physical activity level on the association between motor coordination and body fatness;

2) to analyze the influence of body fatness on the association between motor coordination and physical activity;

3) to examine how much physical activity and body fatness, separately and together, explain motor coordination in adolescent boys.

\section{Material and methods}

Fifty-one boys $(\mathrm{n}=51)$ from a full time public school were recruited to participate in this study. A power analysis conducted with G*POWER 3.1 (Universitat Kiel, Germany) revealed that this sample size was appropriate for a power analysis of $0.80(\beta=0.20)$, an $\alpha=0.05$ and an effect size from moderate to large. The means and standard deviations (SD) of age, body mass and stature of participants are, respectively, 13.8 years $( \pm 0.6), 49.4 \mathrm{~kg}$ $( \pm 12.0)$ and $1.61 \mathrm{~m}( \pm 0.1)$. Students regularly enrolled in the school aged between 12 and 14 years old, without injury or disease that could affect the motor performance, were included in this investigation. Ethical approval for this study was obtained from the University Ethics Committee and parental consent and child assent were obtained prior to participation in the research.

All measurements were performed by a single expert assessor. The physical activity questionnaire was administered within the classroom. Anthropometry and motor coordination tests were administered in a school gymnasium during the school day and lasted approximately $25 \mathrm{~min}$ per participant. Participants were assessed according to their classroom group within a period of one week.
Body mass was measured to the nearest $0.1 \mathrm{~kg}$ using an electronic scale with participants wearing their school uniform. Standing height was measured while unshod with a meter wall to the nearest $0.1 \mathrm{~cm}$. Triceps and gastrocnemius skinfold thickness were measured using a skin caliper, and the mean values were converted to percent (\%) body fat using the Slaughter equation [18].

The Physical Activity Questionnaire for Older Children (PAQ-C), a valid [13] and self-administered 7-day recall instrument, was used to assess general levels of physical activity of participants. The PAQ-C is appropriate for elementary school-aged children approximately between 8 and 14 years old who are currently in the school system and have recess as a regular part of their school week. The summary score from the PAQ-C is the average of the sum of the nine item questions, each scored on a five-point scale. Before using the PAQ-C, it was translated and crossculturally adapted into the language of participants, whose mother tongue was not English, through a structured method using forward-translation and back-translation procedures [2]. The psychometric properties of the new version of PAQ-C reached an adequate level (Cronbach's $\alpha=0.85-0.87$, intraclass correlation coefficient $=0.90$ ).

Motor coordination level was assessed with the KTK by a single trained assessor. The KTK is a reliable a nd valid instrument [12] for middle school-aged children and consists of four test items: (1) walking backwards along balance beams of decreasing width; (2) one-legged hopping over an obstacle, formed by an increasing pile of pillows; (3) two-legged jumping sideways across a wooden slat for $15 \mathrm{~s}$ as quickly as possible; and (4) moving sideways on wooden boards lasting $20 \mathrm{~s}$ as many times as possible. All four scores were gender - and age-adjusted. The motor coordination level for each participant was derived from the sum of the four age-adjusted scores obtained in the tests.

Descriptive statistics were determined for all variables. The Kolmogorov-Smirnov test confirmed acceptable normality of the data distribution of entire sample $(n=51$ participants). The Kruskal-Wallis test was performed to compare levels of motor coordination, physical activity, and body fatness according to age $(12,13$, and 14 years old). Pearson's correlation coefficient was used to examine the bivariate relationships among motor coordination, body fatness and physical activity level. Partial correlation was used to examine the association between motor coordination and body fatness, controlling for physical activity level. Also, partial correlation was used to examine the association between motor coordination and physical activity, controlling for body fatness. The following categories were adopted for classifying the strength of the correlation coefficient: little or inexistent $(r \leq 0.25)$, weak $(0.25<\mathrm{r}<0.50)$, moderate $(0.50 \leq \mathrm{r}<0.70)$, strong $(0.70 \leq \mathrm{r}<0.90)$ and very strong $(\mathrm{r} \geq 0.90)$. Regression analysis was used to show how much physical activity and 
body fat percentage, separately and together, can explain motor coordination. A significance level of $5 \%(\alpha=0.05)$ was adopted in all statistical tests. Data analysis was executed using IBM SPSS ${ }^{\circledR}$ software ver. 22.0.

\section{Results}

Descriptive data, including mean $( \pm \mathrm{SD})$ and confidence interval (CI) of motor coordination, physical activity level and body fatness of participants $(\mathrm{n}=51)$ were, respectively, as follows: $95.2 \pm 15.9$ and CI: $90.7-99.7 ; 3.0 \pm 0.7$ and CI: $2.8-3.2 ; 16.0 \pm 7.1$ and CI: 14.0-18.1. Descriptive data for these variables stratified by age are displayed in Figures 1-3.

Among 51 participants, seven $(n=7)$ were 12 years, eighteen $(n=18)$ were 13 years and twenty six $(n=26)$ were 14 years old. There were no significant differences $(\mathrm{p}>0.05)$ in motor coordination, physical activity and body fat percentage among age groups (Figures 1-3).

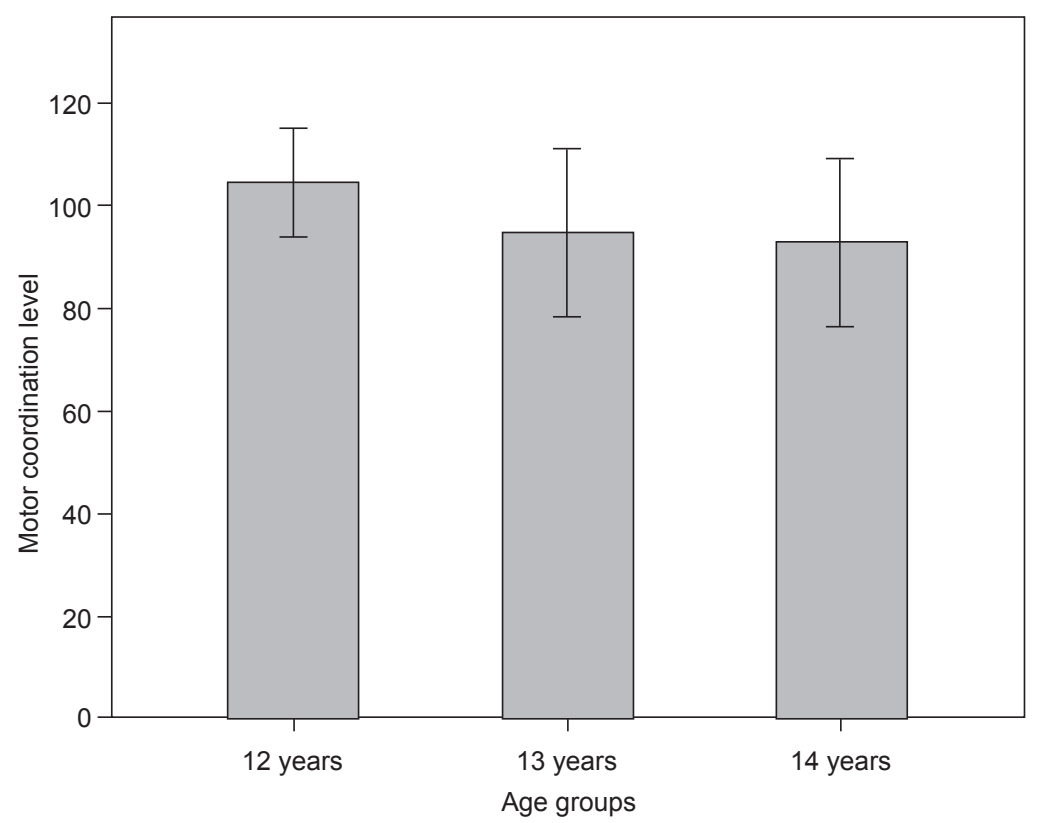

Fig. 1. Mean ( \pm standard deviation) motor coordination level (nondimensional measure) across age groups. Error bar denotes standard deviation

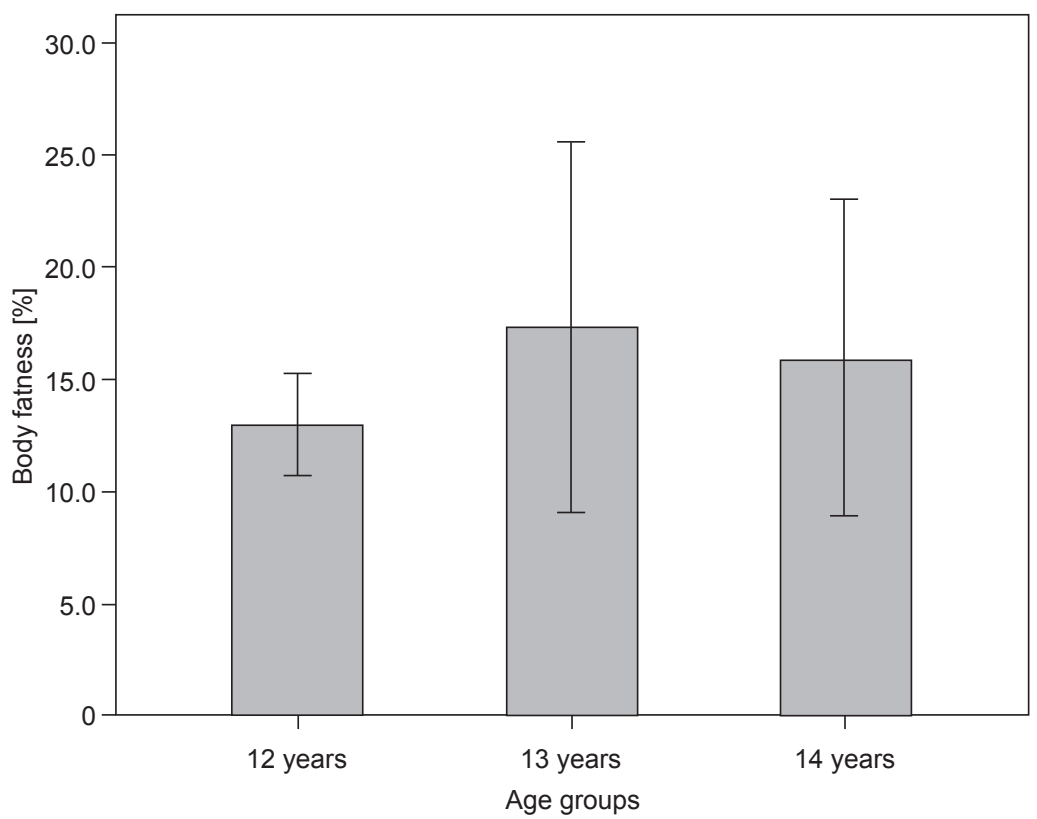

Fig. 2. Mean ( \pm standard deviation) body fatness (\%) across age groups. Error bar denotes standard deviation 


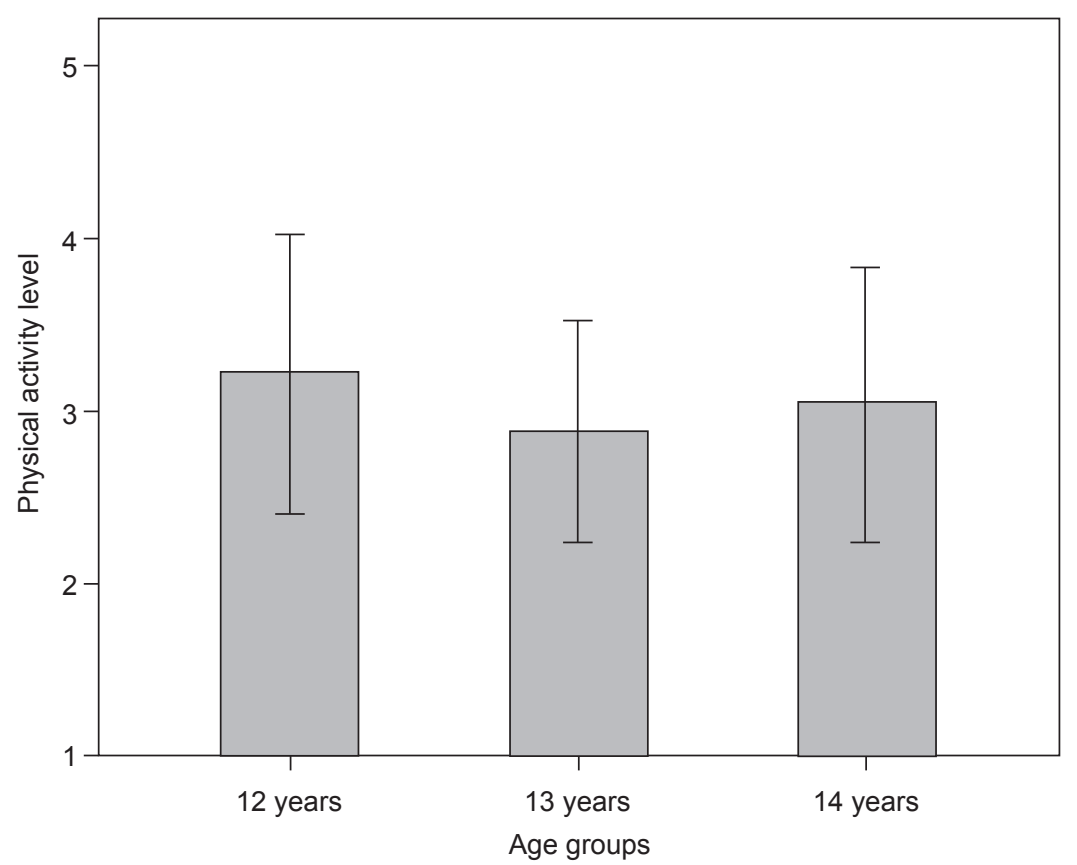

Fig. 3. Mean ( \pm standard deviation) physical activity level (nondimensional measure) across age groups. Error bar denotes standard deviation

Then, correlational analyses in this study were performed using the sample as a whole $(\mathrm{n}=51)$.

Motor coordination level was weakly correlated with the physical activity level and body fatness. Also, partial correlation analyses revealed that these correlations were not statistically significant when controlled for body fatness and physical activity, respectively. Both Pearson and partial correlations coefficient are described in Table 1. In addition, the bivariate correlation between body fat and physical activity level was not significant $(\mathrm{r}=-0.227$; $\mathrm{p}=0.110)$.

Table 1. Pearson and partial correlations coefficients among motor coordination, physical activity and \% body fat in adolescent boys $(n=51)$

\begin{tabular}{lcc}
\hline & Bivariate & Partial \\
\hline $\begin{array}{l}\text { Motor coordination and } \\
\text { physical activity }\end{array}$ & $\begin{array}{c}0.300 \\
(\mathrm{p}=0.032)\end{array}$ & $\begin{array}{c}0.250^{\dagger} \\
(\mathrm{p}=0.080)\end{array}$ \\
\hline $\begin{array}{l}\text { Motor coordination and } \\
\% \text { body fat }\end{array}$ & $\begin{array}{c}-0.297 \\
(\mathrm{p}=0.032)\end{array}$ & $\begin{array}{c}-0.247^{\dagger} \\
(\mathrm{p}=0.084)\end{array}$ \\
\hline
\end{tabular}

$\dagger$ - partial correlation controlled for \% body fat; $\ddagger$ - partial correlation controlled for physical activity.
Multiple regression analysis revealed that body fat and physical activity, together, explained $11.0 \%$ of the variance (adjusted $R^{2}=0.110 ; \mathrm{F}=4.084, \mathrm{p}=0.023$ ) in the motor coordination level. However, body fat and physical activity, separately, did not significantly predict motor coordination level (Table 2).

\section{Discussion}

This study analyzed the three-way interplay of motor coordination, body fatness and physical activity in adolescent boys. The results suggested that body fatness can influence the associations between motor coordination and physical activity level. Also, physical activity level influenced the associations between motor coordination and body fatness in adolescent boys. Finally, body fatness and physical activity together explained $11 \%$ of variance in the motor coordination level.

Correlation analysis revealed that physical activity level was negatively, but insignificantly, associated with body fat percentage. Although these variables tend to be negatively associated $[1,19]$, it is important to highlight that

Table 2. Coefficients $(\beta)$ of the independent variables in the multiple linear regression model

\begin{tabular}{lcccc}
\hline Independent variable & $\beta$ (unstandardized) & $\beta$ (standard error) & $\beta$ (standardized) & Significance of $\beta(\mathrm{t}$ test) \\
\hline \%body fat & -0.540 & 0.306 & -0.242 & 0.084 \\
Physical activity & 5.264 & 2.942 & 0.245 & 0.080 \\
\hline
\end{tabular}


not all individuals with a high body fat percentage have low levels of physical activity. Therefore, these findings confirm the assumption that having elevated body fatness and increased levels of physical activity are not mutually exclusive conditions.

In line with previous findings $[3,5,6,8,10,20]$, the results of this study indicated that motor coordination level and body fatness are negatively associated in adolescent boys. A plausible explanation for these results is based on biomechanical principles. That is, the higher the amount of body mass of an individual, the higher is the inertial moment, as well as the higher the mechanical work required to perform motor tasks, especially those which demand weight-bearing. This explanation is known as the weight-bearing hypothesis [7]. Therefore, our findings were expected, because the test used to assess motor coordination in this study, the KTK, involves weight-bearing tasks, such as skipping and moving quickly.

Overall, previous studies suggest that levels of motor coordination and physical activity level are positively associated $[11,14,15,17,21]$. Our findings corroborated these previous results. In this regard, Stodden et al. [19] proposed a conceptual model in which the level of physical activity contributes to the development of motor competence, and vice versa. Therefore, it was expected that levels of motor coordination and physical activity would have a positive correlation in adolescent boys.

The correlation between motor coordination and physical activity was not significant when controlled for body fatness in adolescent boys. Also, the correlation between motor coordination and body fatness was not significant when controlled for physical activity. These results indicate that body fatness influences the association between motor coordination and physical activity. Further, in line with a previous study [3], these results suggest that physical activity influence the association between motor coordination and body fatness in adolescents [3]. Although it is well documented that motor coordination level is negatively associated with body fatness $[3,5,6,8,10,20]$ and positively associated with physical activity $[11,14$, $17,21]$, there are other variables that can interfere with this relationship. In this regard, D'Hondt et al. [8] argued that the negative association between motor coordination and body fatness is mediated by several alternative, and possibly complementary mechanisms.

Multiple regression analysis revealed that body fat and physical activity, separately, did not significantly predict motor coordination level. These findings were expected due to weak bivariate correlations found in the associations of motor coordination with body fat and physical activity. On the other hand, body fat and physical activity, together, explained $11 \%$ of the variance in the motor coordination level. Altogether, these findings suggest that body fat and physical activity are complementarily associated with motor coordination in adolescent boys.

First, it is important to highlight that it is difficult to make comparisons across investigations due to differences related to study designs. However, it seems plausible that the differences in the results between studies may be related to the magnitude of the physical activity level and body fatness of participants. As previously argued [5], the influence of confounding of body fatness and physical activity level on the associations between motor coordination and physical activity, and between motor coordination and body fatness, respectively, may be related to the magnitude of these variables. Additional studies should be performed to test this hypothesis.

The motor coordination level showed by individuals is a complex phenomenon that is associated with factors related to the individual and the constraints of the task and the environment [9]. For example, the individual's body fatness interferes with the mechanical work performed in certain motor tasks, and his motor coordination level also depends on other factors, such as the experienced opportunities with practice throughout the lifespan. In fact, adolescents with higher body fatness may have higher levels of physical activity and motor coordination than their peers with lower body fatness. Thus, physical activity level of an individual can minimize the negative association between body fatness and motor coordination level. Similarly, a high level of body fatness may minimize the positive association between physical activity level and motor coordination. The findings of this present investigation suggest that the study of the association between motor coordination level and health-related attributes should not be restricted to the bivariate analysis between such variables.

The physical activity level may influence the association between motor coordination level and body fatness in adolescent boys. Likewise, body fatness may influence the association between motor coordination and physical activity in adolescent boys. Also, body fat and physical activity together, but not separately, partially explained the variance in the motor coordination level. Therefore, body fat and physical activity seem to be complementarily associated with motor coordination in adolescent boys.

Conflict of interest: Authors state no conflict of interest.

\section{References}

1. Barnett L.M., Lai S.K., Veldman S.L., Hardy L.L., Cliff D.P., Morgan P.J., Zask A., Lubans D.R., Shultz S.P., Ridgers N.D., Rush E., Brown H.L., Okeley A.D. (2016) Correlates of gross motor competence in children and ad- 
olescents: a systematic review and meta-analysis. Sports Med., 46: 1663-1688.

2. Beaton D., Bombardier C., Guillemin F., Ferraz M. (2000) Guidelines for the process of cross-cultural adaptation of self-report measures. Spine, 25: 3186-3191.

3. Chagas D.V., Batista L.A. (2016) Associations between motor coordination and BMI in normal weight and overweight/obese adolescents. J. Hum. Growth Dev., 26: 380-384.

4. Chagas D.V., Batista L.A. (2017) Comparison of health outcomes among children with different levels of motor competence. Hum. Mov., 18: 56-61.

5. Chagas D.V., Batista L.A. (2015) Interrelationships among motor coordination, body fat percentage, and physical activity in adolescent girls. Hum. Mov., 16:4-8.

6. Chagas D.V., Carvalho J.F., Batista L.A. (2016) Do girls with excess adiposity perform poorer motor skills than leaner peers? Int. J. Exerc. Sci., 9: 318-326.

7. D'Hondt E., Deforche B., De Bourdeaudhuij I., Lenoir M. (2009) Relationship between motor skill and body mass index in 5- to 10-year-old children. Adapt. Phys. Activ. $Q$. 26: 21-37.

8. D'Hondt E., Deforche B., Gentier I., De Bourdeaudhuij I., Vaeyens R., Philippeaerts R., Lenoir M. (2013) A longitudinal analysis of gross motor coordination in overweight and obese children versus normal-weight peers. Int. J. Obes., (London), 37: 61-67.

9. Gallahue D., Ozmun J., Goodway J. (2012) Understanding motor development: infants, children, adolescents, adults. 7th edition. McGraw-Hill, New York.

10. Kakebeeke T.H., Lanzi F., Zysset A.E., Arhab A., Messerli-Bürgy N., Stuelb K., Leeger-Aschmann C.S., Schmutz E.A., Meyer A.H., Kriemler S., Munsch S., Jenni O.G., Puder J.J. (2017) Association between body composition and motor performance in preschool children. Obes. Facts, 10: 420-431.

11. Kambas A., Michalopoulou M., Fatouros I., Christoforidis C., Manthou E., Giannakidou D., Venetsanou F., Haberer E., Chatzinikolaou A., Gourgoulis V., Zimmer R. (2012) The relationship between motor proficiency and pedometer-determined physical activity in Young children. Pediatr. Exerc. Sci., 24: 34-44.

12. Kipard E., Shilling F. (2007) Körperkoordinationstest für Kinder. Göttingen, Hogrefe.
13. Kowalski K., Crocker P., Faulkner R. (1997) Validation of the Physical Activity Questionnaire for Older children. Pediatr. Exerc. Sci., 9: 174-186.

14. Laukkanen A., Pesola A., Havu M., Sääkslahti A., Finni T. (2014) Relationship between habitual physical activity and gross motor skills is multifaceted in 5- to 8-year-old children. Scan. J. Med. Sci. Sports, 24: 102-110.

15. Lopes V.P., Rodrigues L.P., Maia J.A.R., Malina R.M. (2011) Motor coordination as predictor of physical activity in childhood. Scand. J. Med. Sci. Sports., 21: 663-669.

16. Morrison K., Bugge A., El-Naaman B., Eisenmann J., Froberg K., Pfeiffer K., Andersen L.B. (2012) Inter-relationships among physical activity, body fat, and motor performance in 6- to 8-year-old Danish children. Pediatr. Exerc. Sci., 24: 199-209.

17. Robinson L.E., Stodden D.F., Barnett L.M., Lopes V.P., Logan S.W., Rodrigues L.P., D'Hondt E. (2015) Motor competence and its effect on positive developmental trajectories of health. Sports Med., 45: 1273-1284.

18. Slaughter M., Lohman T., Boileau R. (1988) Skinfold equations for estimation of body fatness in children and youth. Hum. Biol., 60: 709-723.

19. Stodden D.F., Goodway J.D., Langendorfer S.J., Roberton M., Rudisill M., Garcia C., Garcia L. (2008) A developmental perspective on the role of motor skill competence in physical activity: an emergent relationship. Quest., 60: 290-306.

20. Vandendriessche J., Vandorpe B., Coelho-e-Silva M., Vaeyens R., Lenoir M., Lefevre J., Philippaerts R. (2011) Multivariate association among morphology, fitness, and motor coordination characteristics in boys age 7 to 11 . Pediatr. Exerc. Sci., 23: 504-520.

21. Wrotiniak B., Epstein L., Dorn J., Jones K., Kondilis V. (2006) The relationship between motor proficiency and physical activity in children. Pediatrics, 118: e1758-e1765.

\section{Received 15.04.2019 \\ Accepted 24.07.2019}

(C) University of Physical Education, Warsaw, Poland 